\title{
REVIEWS
}

\section{LUDO ERGO SUM: DOES GAME PLAYING REVEAL YOUR PERSONALITY?}

\author{
Quantifying Individual Player Differences \\ Giel van Lankveld \\ PhD Thesis, Tilburg University 2013, 210 pp. \\ TiCC Ph.D.Series 25
}

Reviewed by Dap Hartmann

\begin{abstract}
Can computer games be used to determine a person's psychological profile? That is an intriguing question with profound consequences if the answer turns out to be affirmative. One look at this YouTube video: http://www.youtube.com/watch? $\mathrm{v}=\mathrm{PbcctWbC8Q0}$ subtly hints that there is reason to believe that such a correlation exists. Giel van Lankveld seeks to answer this question in his PhD thesis 'Quantifying Individual Player Differences', although he states it a bit more careful and cautious: "To what extent are games an appropriate means for measuring the differences between individuals based on psychological theories?" From a game developer's perspective, this is an interesting question because it may help increase the control and understanding of a player's experience while playing a game, and it can also aid in adapting game content to suit individual players. However, there could be a more important spinoff from this research, namely that computer games can be used as a cheap and simple way of psychologically profiling people. Van Lankveld carefully avoids that particular can of worms, but this statement in the colophon of his thesis indicates that it could indeed be an interesting offshoot: "This research has been partly funded by the project KIM (Kennis in Modellen) of the Korps Landelijke Politie Diensten (KLPD), supported by the Dutch Ministry of Security and Justice." In plain English it says that the thesis has been sponsored in part by the 'Knowledge in Models' project of the Dutch National police force.
\end{abstract}

The aim of this research is to use psychological personality theories and models to extend the most common methods of modeling players in computer science: user, opponent and player models. Van Lankveld investigated three aspects: (1) using psychological models to create player models, (2) automatically adapting games to players' skills and personalities, and (3) using games to generate personality profiles. The approach to incorporating psychological modeling in player profiling appears to be rigorous, with no less than 19 definitions, although some are a little puzzling (if not cyclically redundant) to non-experts such as I: "Definition 2.19 (Personality theory) Personality is a class of psychological theory that is concerned with personality." To profile the people who participated in the experiments, Van Lankveld used four different questionnaires: a general questionnaire with questions such as "How much do you agree with the statement that you love to drive cars", an incongruity questionnaire containing 26 items to assess the emotions predicted by the incongruity theory in five categories (boredom, frustration, pleasure, concentration, and curiosity), a personality questionnaire consisting of 240 questions to determine five domains (Openness to Experience, Conscientiousness, Extraversion, Agreeableness, and Neuroticism - collectively known under the acronym OCEAN) and an emotional questionnaire that measures both positive and negative emotions as well as attention and interest. In addition, two observational techniques were used: Player Behavior Logging (PBL) which records events that occur during game playing ("Moving toward an aggressive looking monster has a meaning different from moving toward an innocent looking small child"), and Rated Video Observation (RVO) in which a video recording of an interview with a test subject is viewed and rated on personality variables by several observers.

Van Lankveld addresses his main problem statement ("To what extent are games an appropriate means for measuring the differences between individuals based on psychological theories?") by five research questions. Each research question is the subject of an entire chapter which explains the background, the theoretical framework and the methodology used to answer that question. I am no expert in psychology, but from a computer science point of view it appears a bit curious to investigate each research question with a different group of test subjects. Why not keep one and the same group of people? For example, to answer research question 1 "To what extent are games suitable for measuring incongruity?" 24 test subjects between the ages of 16 and 31 years played a game called GLOVE which is an updated version of the classic game GAUNTLET. Neither game was familiar to me, but fortunately Van Lankveld explains the purpose and the rules of GLOVE. Each test subject played the game four times: once as a trial run and once at each of the three difficulty settings (easy, balanced, hard). After each game, a questionnaire with 26 items which measure five emotions (boredom, 
frustration, pleasure, concentration, and curiosity) is filled out by each player. Despite the relatively small group of test subjects, Van Lankveld presents the main conclusion as highly significant: "frustration increases with difficulty, while the pleasure value remains roughly the same for easy and balanced difficulty, but drops drastically for hard difficulty." To answer the next research question "To what extent can games be used to measure complex psychological traits such as extraversion?" Van Lankveld uses a different group of test subjects. In this experiment, 39 test subjects (20 males and 19 females) aged between 18 and 43 years, first played a game and then completed the personality questionnaire and an additional questionnaire which is rather vaguely described as "containing topics of possible relevance to the experiment". The game used in this trial was NEVERWINTER NigHTS, a top-down role-playing game into which a special scenario was introduced that contains 21 in-game elements based on the personality questionnaire. 30-40 minutes were needed to complete the game, while each of the questionnaires required about 10 minutes to fill out. The conclusion of this experiment is that five of the in-game elements had a significant correlation to the extraversion scores in the personality test. In other words, the answers to the extraversion-related questions in the questionnaire were reflected in respondents' behavior while playing the game.

Van Lankveld does not explain why the experiments were done with different test groups, nor does he address the question whether or not that influences the results and the conclusions. Another issue that raises some questions is that GLOVE is a game in which the player has to fight and kill enemies using weapons. And while NEVERWINTER NIGHTS appears innocent (a little girl asks the player to deliver a letter to the king) the brief description provided mentions that "the road to the king is filled with obstacles and encounters". While the original game of NEVERWINTER NIGHTS certainly contains violent elements (assassins, battles), it is undisclosed whether violence is involved in the new scenario. Furthermore, to answer research question 5 "To what extent can our models of personality in games be validated in different games?", the test subjects (yet another population, this time 36 students - 18 males, 17 females, and 1 'unknown' - between the ages of 18 and 27) played the game FALLOUT 3, which is extremely violent. I just wonder whether this choice of violent games affected the outcome of the experiment. For example, one might argue that people who enjoy playing games in which opponents are destroyed in very graphically explicit ways have different personalities from people who prefer to play more peaceful games such as SOKOBAN or TETRIS, and whether this influences the general conclusions of this research. I assume that the second promotor in the thesis committee, Arnoud Arntz, professor of clinical psychology and neuroscience at Maastricht University, approved the experimental setups used in this research. According to the university website, Arntz's fields of interest are "the experimental study of pain, anxiety disorders and personality disorders, with a special interest in investigating information processing in these areas" which appears to be highly relevant to the research described in Van Lankveld's thesis.

Van Lankveld concludes that "all methods used would benefit from a larger test sample." Again, no word on the use of different populations of test subjects in the various experiments. The answer to the problem statement is stated as follows: "we consider games to be an appropriate means for investigating individual differences based on psychological theories given the fact that we have found both the theory-driven as well as the data-driven correlation results and regression results for personality in games." I must admit that I am a bit disappointed by the only tangible result from all these experiments, namely that there are "relationships between emotions (frustration and pleasure) and game difficulty as predicted by incongruity theory." In other words, people get frustrated by games that are hard to play, and this gives them little pleasure. It is hard for me to separate these two emotions, as I cannot think of anything that is frustrating but nevertheless gives pleasure. Hopefully this conclusion is significant in the field of psychology, because I doubt it has any impact on artificial intelligence.

The full thesis can be downloaded as a PDF document from http://arno.uvt.nl/show.cgi?fid=129313 Unfortunately, it does not include the cover. Not that the cover is particularly beautiful or otherwise essential to the thesis, but it is just my sense of aesthetics that demands a complete electronic version of a book. How else can we judge a book by its cover? 